\title{
Argon-hydrogen plasma jet investigated by active and passive spectroscopic means
}

\author{
R. F. G. Meulenbroeks, A. J. van Beek, A. J. G. van Helvoort, M. C. M. van de Sanden, and D. C. Schram \\ Eindhoven University of Technology, Department of Physics, P.O. Box 513, 5600 MB Eindhoven, the Netherlands
}

(Received 23 August 1993)

\begin{abstract}
A supersonically expanding argon cascaded arc plasma, with different amounts of hydrogen added $(0$, 0.7 , and $1.4 \mathrm{vol} \% \mathrm{H}_{2}$ ), was studied using Thomson-Rayleigh scattering and optical emission spectroscopy. With hydrogen added, the electron density profile as a function of the distance from the onset of the expansion shows a large extra ionization loss (compared to the pure argon case), especially after the stationary shock front. This anomalous loss of ionization is attributed to molecular processes, such as associative charge transfer between $\mathrm{Ar}^{+}$and $\mathrm{H}_{2}$, and dissociative recombination of the resulting $\mathrm{ArH}^{+}$ molecular ion. Spatially resolved emission spectroscopy shows that in the expansion the radial hydrogen emission profiles are broader than the argon profiles. The addition of hydrogen appears to change the characteristic shock behavior of pure argon. For both the argon and the hydrogen system it is shown that the uppermost levels are in Saha equilibrium with their adjacent continuum. The measurements are in agreement with the view that association of hydrogen atoms at the vessel walls results in a reentry flow of hydrogen molecules in the plasma beam.
\end{abstract}

PACS number(s): 52.70.Kz, 52.25.Rv, 52.40.Hf

\section{INTRODUCTION}

The fundamental study of plasmas, expanding out of a cascaded arc thermal plasma source, is important in view of the use of these plasmas for ultrafast deposition of $a: \mathrm{Si}-\mathrm{H}, a: \mathrm{C}-\mathrm{H}$, graphite, and diamond thin films [1-3]. In the past, pure argon expanding plasmas have been studied extensively, especially the stationary shock front, occurring a few centimeters after the onset of the supersonic expansion. This research has resulted in a good understanding of expanding plasmas in pure argon [4-6]. The next step towards deposition plasmas is the study of argon plasmas with small amounts of simple molecules added, e.g., $\mathbf{H}_{2}$. The research on argon-hydrogen plasmas is important for another reason: these plasmas may be used as hydrogen atom and hydrogen negative or positive ion sources. In the following, an experimental study of argon-hydrogen plasma jets is presented.

\section{EXPERIMENT}

\section{A. The cascaded arc experiment}

In Fig. 1, the experiment is sketched [5]. The cascaded arc plasma source consists of three cathodes, three electrically isolated copper plates, and an end anode plate. All the parts are water cooled [7]. The arc channel has a diameter of $4 \mathrm{~mm}$. The arc produces a thermal plasma at subatmospheric pressure, characterized by a relatively high heavy particle temperature $\left(T_{h} \approx T_{e} \approx 1 \mathrm{eV}\right)$ and high electron densities $\left(10^{22}-10^{23} \mathrm{~m}^{-3}\right)$.

This plasma is allowed to expand onto a low-pressure background, creating a supersonically expanding jet, ending in a stationary shock. The low-pressure region consists of a heavily pumped (maximum 700 liters/s) stainless-steel vessel. After the shock, axial velocities are subsonic (i.e., around $600 \mathrm{~m} / \mathrm{s}$ ). The experimental condi- tions for all the following experiments are $I_{\mathrm{arc}}=45 \mathrm{~A}$, $V_{\text {arc }}=105 \mathrm{~V}$, background pressure $p_{\text {back }}=40 \mathrm{~Pa}$, argon gas flow $=3.5$ SLM (standard liter per minute).

Hydrogen can be added in small amounts to the flow before it enters the cascaded arc. In the experiments, the hydrogen flows chosen were 25 SCCM (standard cubic centimeters per minute) and $50 \mathrm{SCCM}$, resulting in $\mathbf{H}_{2}$ fractions of 0.7 and $1.4 \mathrm{vol} \%$ entering the arc.

\section{B. Diagnostics}

\section{Thomson-Rayleigh scattering}

Figure 2 shows the combined Thomson-Rayleigh diagnostic, which is described in detail elsewhere [5]. The frequency doubled Nd:YAG (yttrium aluminum garnet) laser (Quanta Ray DCR 11) radiation at $532 \mathrm{~nm}$ is focused onto the plasma. The scattered radiation is observed under a $90^{\circ}$ angle, and analyzed by a polychromator and an image intensified photodiode array. The stray light level is reduced substantially by placing the laser dump far away, outside the vessel. The analysis of the scattered signal gives accurate and spatially resolved

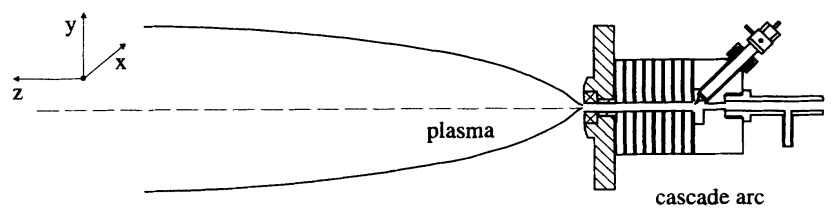

FIG. 1. The cascaded arc experiment, a thermal plasma at atmospheric pressure is created in a continuously operated dc arc, consisting of three cathodes, three electrically isolated copper plates, and an anode plate. All these parts are water cooled. The plasma expands into a low-pressure region, creating a supersonically expanding plasma jet. 


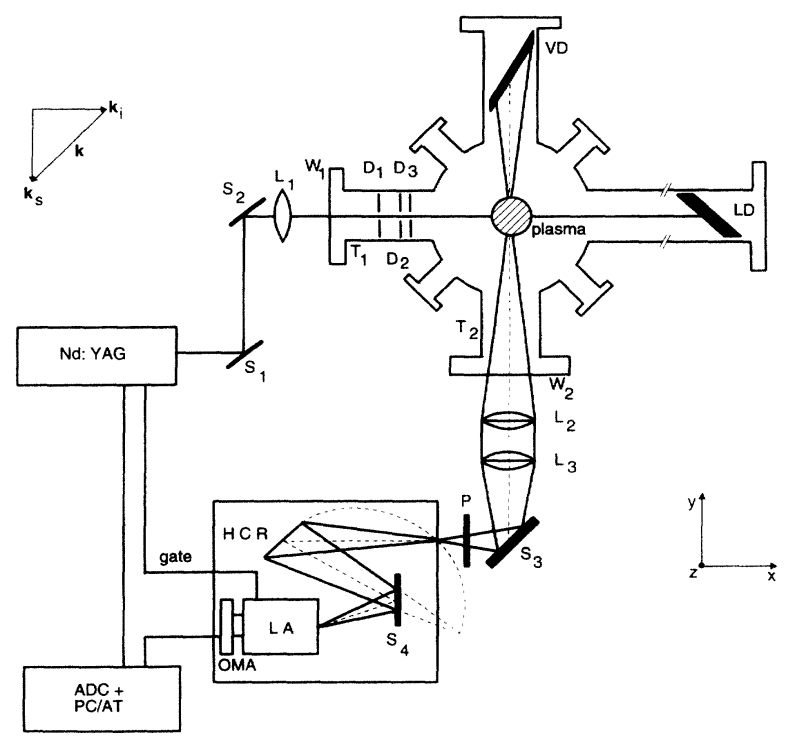

FIG. 2. The Thomson-Rayleigh diagnostic, frequency doubled Nd:YAG radiation is focused onto the plasma by a system of two dichroic mirrors $\left(S_{1}\right.$ and $\left.S_{2}\right)$ and a lens $L_{1}$. The stray light level is reduced by placing the laser dump (LD) far away (i.e., $2 \mathrm{~m}$ ), and installing several diaphragms $D_{1}-D_{3}$. The scattered radiation is transmitted through a system consisting of lenses $L 2$ and $L 3$ and a mirror $S 3$. Detected radiation is dispersed by a hollow concave grating (HCR), amplified by a gated light amplifier (LA), and detected by an optical multichannel analyzer (OMA). The data are processed by a personal computer (PC/AT), using an analog-to-digital convertor (ADC).

(scattering volume $\approx 0.25 \mathrm{~mm}^{3}$ ) information about the electron density, electron temperature, and the neutral particle density in the plasma. The plasma source can be moved along the $z$ axis without significantly changing the plasma, making axial scans possible while keeping the diagnostics fixed.

\section{Optical emission spectroscopy (OES)}

Plasma radiation is measured with an optical emission spectroscopy experiment (Fig. 3 [4]). A Czerny-Turner type monochromator (Jarrell-Ash $0.5 \mathrm{~m}$ ) is used in combination with a Peltier-cooled photomultiplier tube (RCA $31034)$ to analyze the plasma radiation. Entrance and exit slits of 25 and $100 \mu \mathrm{m}$, respectively, are used and result in a spectral resolution of $0.16 \mathrm{~nm}$. The output of the photomultiplier is amplified and analyzed by a photoncounting system. Lateral plasma scans are possible by moving a step motor-driven rotating mirror in the system. The photon-counting data processing and step motor control are implemented in a modular, M68000 processor-based, laboratory computer system. A low wavelength cutoff filter was used for wavelengths above $500 \mathrm{~nm}$ to eliminate second-order radiation. The OES system was calibrated in an absolute way using a tungsten ribbon lamp placed at the plasma location in the vessel.

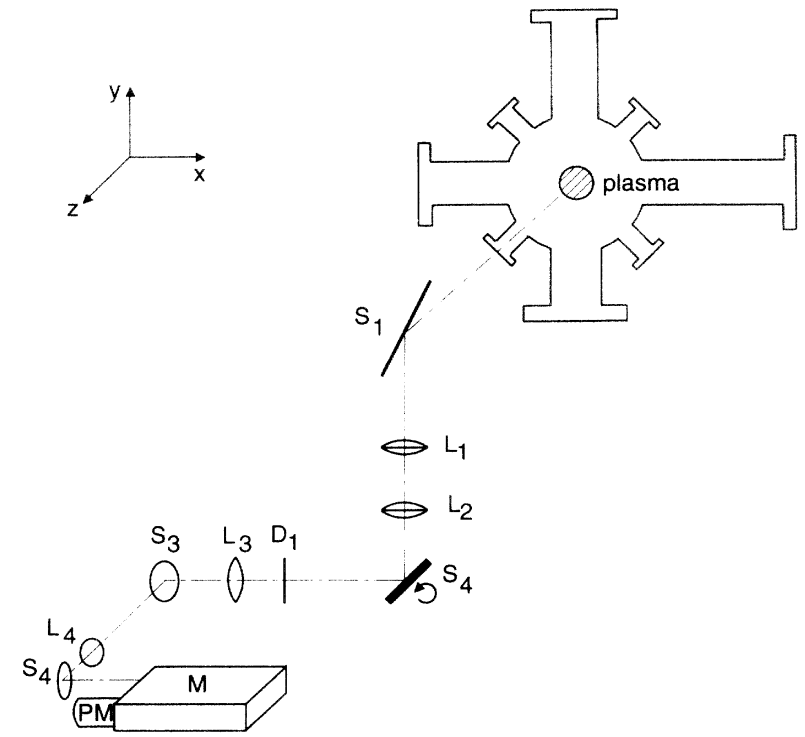

FIG. 3. The optical emission spectrometer plasma radiation is transmitted through a system of mirrors $\left(S_{1}-S_{4}\right)$ and glass lenses $\left(L_{1}-L_{4}\right)$. Mirror $S_{2}$ can be rotated in order to perform lateral plasma scans. A second-order filter can be inserted in front of lens $L_{4}$ (for wavelengths above $500 \mathrm{~nm}$ ). A CzernyTurner monochromator $(M)$ analyzes the radiation with a resolution of $0.16 \mathrm{~nm}$. A cooled photomultiplier (PM) detects the transmitted photons.

\section{Measurements}

\section{Thomson-Rayleigh scattering}

Local measurements of the electron density, electron temperature, and neutral particle density are carried out locally at different distances from the expansion nozzle, at the plasma jet axis. Because of an effective stray light reduction and an accurate fit procedure, relative errors in these measured quantities are low: about $5 \%, 15 \%$, and $20 \%$, respectively [5]. The neutral particle density, determined from the Rayleigh scattered signal, is about equal to the argon heavy particle concentration, as the hydrogen seed-in flow is very small. The measurements are performed for $0,0.7$, and $1.4 \mathrm{vol} \% \mathrm{H}_{2}$ admixed in argon.

\section{Optical emission spectroscopy}

Lateral scans of the plasma are performed for 13 argon neutral lines and the first four hydrogen Balmer lines. Usually, every lateral scan consists of 80 measurements, covering a lateral range of $100 \mathrm{~mm}$. These lateral measurements are converted to radial profiles by a fast Abelinversion procedure using filtered back projection [8]. After correction for the efficiency of the system (using the ribbon lamp calibration), these radial profiles can be converted into radial profiles of the absolute level population per statistical weight. The lines, selected for the light emission measurements, with relevant data [9], are presented in Table $\mathbf{I}$.

The OES measurements are performed at three axial 
TABLE I. The argon and hydrogen lines selected for the OES measurements. $E_{p}$ (in eV) is the energy measured from the ground state, $I_{p}\left(\mathrm{in} \mathrm{eV}\right.$ ) is the ionization energy of the level $E_{i}-E_{p}, \lambda$ gives the wavelength of the transition in $\mathrm{nm}, g_{p}$ is the degeneracy of the upper level, and $A_{p q}$ is the transition probability (in $10^{8} \mathrm{~s}^{-1}$ ). The accuracies are the relative errors in the $A_{p q}$ 's.

\begin{tabular}{|c|c|c|c|c|c|c|}
\hline Transition & $\lambda$ & $E_{p}$ & $I_{p}$ & $g_{p}$ & $A_{p q}$ & Accuracy $(\%)$ \\
\hline \multicolumn{7}{|c|}{ Argon } \\
\hline $4 p \rightarrow 4 s$ & 811.5 & 13.07 & 2.69 & 7 & 0.366 & 25 \\
\hline $4 p \rightarrow 4 s$ & 763.5 & 13.17 & 2.59 & 5 & 0.274 & 25 \\
\hline $4 p^{\prime} \rightarrow 4 s$ & 696.5 & 13.32 & 2.44 & 3 & 0.067 & 25 \\
\hline $4 p^{\prime} \rightarrow 4 s^{\prime}$ & 750.4 & 13.48 & 2.275 & 1 & 0.472 & 25 \\
\hline $5 p \rightarrow 4 s$ & 416.4 & 14.52 & 1.239 & 3 & 0.00295 & 25 \\
\hline $5 p \rightarrow 4 p$ & 420.1 & 14.57 & 1.185 & 7 & 0.0103 & 25 \\
\hline $4 d \rightarrow 4 p$ & 693.8 & 14.69 & 1.065 & 1 & 0.0321 & 25 \\
\hline $6 s \rightarrow 4 p$ & 703.0 & 14.84 & 0.915 & 5 & 0.0278 & 25 \\
\hline $4 d^{\prime} \rightarrow 4 p$ & 591.2 & 15.00 & 0.755 & 3 & 0.0105 & 25 \\
\hline $7 s \rightarrow 4 p$ & 588.9 & 15.18 & 0.575 & 5 & 0.0134 & 25 \\
\hline $5 d^{\prime} \rightarrow 4 p$ & 518.8 & 15.30 & 0.456 & 5 & 0.0138 & 25 \\
\hline $8 s \rightarrow 4 p$ & 506.0 & 15.32 & 0.235 & 9 & 0.0039 & 50 \\
\hline $7 d^{\prime} \rightarrow 4 p^{\prime}$ & 531.8 & 15.63 & 0.125 & 7 & 0.0027 & 50 \\
\hline \multicolumn{7}{|c|}{ Hydrogen } \\
\hline $3 \rightarrow 2$ & 656.3 & 12.09 & 1.507 & 18 & 0.4410 & 1 \\
\hline $4 \rightarrow 2$ & 486.1 & 12.75 & 0.847 & 32 & 0.0841 & 1 \\
\hline $5 \rightarrow 2$ & 430.4 & 13.06 & 0.519 & 50 & 0.0253 & 1 \\
\hline $6 \rightarrow 2$ & 410.2 & 13.22 & 0.378 & 72 & 0.0097 & 1 \\
\hline
\end{tabular}

positions, at distances of 20,40 , and $70 \mathrm{~mm}$ from the nozzle, for the pure argon case and for the two hydrogenargon mixtures. The accuracy of OES results is mainly determined by the inaccuracies in the transition probabilities (Table I), which amount to $25-50 \%$ for argon. Furthermore, plasma reproducibility errors as well as calibration errors have to be taken into consideration: \pm 20 and $10 \%$, respectively.

\section{RESULTS}

\section{A. Thomson-Rayleigh scattering results}

The results of the axial scans are presented in Fig. 4. The neutral density behavior is equal (within the margin of error) for all three conditions. Adding a small amount of hydrogen, therefore, does not influence the general structure of the expansion, as expected for these low admixtures. The expansion consists of a supersonic part $(z \approx 0-40 \mathrm{~mm})$, a shock region $(z \approx 40-70 \mathrm{~mm})$, and a subsonic relaxation region (after $z=70 \mathrm{~mm}$ ). The electron temperature is also nearly independent of the hydrogen admixture. The data scattering in this case, however, makes it difficult to draw conclusions about possible slight changes in temperature behavior. The electron density is severely influenced by adding such a small amount of hydrogen, especially in the subsonic relaxation region. To make this more clear, Fig. 5 gives the electron density as normalized to that of the pure argon case. The data show a somewhat lower electron density right from the onset of the expansion. The ratio remains constant for the first $70 \mathrm{~mm}$, but starts to decrease rapidly after the shock. This decrease turns out to be about twice as fast when the amount of admixed hydrogen is doubled.

\section{B. Optical emission spectroscpy results}

As was pointed out above, proper calibration of the OES system makes possible the calculation of radial profiles of the absolute level population per statistical weight $n_{p} / g_{p}$. These profiles turned out to be quite congruent both for the different argon lines and for the different hydrogen lines. The differences between the two species, however, are substantial, so we concentrate on one argon and one hydrogen line (Ar I $696.5 \mathrm{~nm} ; \mathrm{H} \mathrm{I}$ $656.3 \mathrm{~nm})$.

Figure 6, then, shows these radial $n_{p} / g_{p}$ profiles for the 696.5 Ar I transition ( $4 p^{\prime}$ level, Table I), at three $z$ positions and for 0, 0.7, and $1.4 \mathrm{vol} \%$ hydrogen added. Especially the behavior at $z=40 \mathrm{~mm}$ is interesting, as the characteristic shock dip at $r=0$ seems to disappear when more hydrogen is added, a phenomenon observed for all argon lines measured. The hydrogen $\mathrm{H} \alpha[p$ (principle quantum number) $=3$ ] radial profiles are shown in Fig. 7 for the same two hydrogen admixture conditions. These hydrogen profiles prove to be substantially wider than the corresponding argon profiles. This is even more apparent in Fig. 8, where the $n_{p} / g_{p}$ profiles for argon and hydrogen have been normalized.

The $n_{p} / g_{p}$ profiles of highly excited levels can be used to obtain the ion concentration ratio $n_{\mathrm{Ar}^{+}} / n_{\mathrm{H}^{+}}$by a method utilized by Meeusen et al. [10]. The argument starts by assuming a Saha equilibrium between the uppermost excited levels and their adjacent continuum: 


$$
\frac{n_{s}+n_{e}}{(n / g)_{s, p}}=C u_{s}+T_{e}^{3 / 2} \exp \left[-\left(E_{i}-E_{p}\right) / k T_{e}\right] .
$$

The subscript $s$ is used to indicate one of the species Ar or $\mathrm{H}$, whereas a superscript " + " denotes the correspond-

(a)

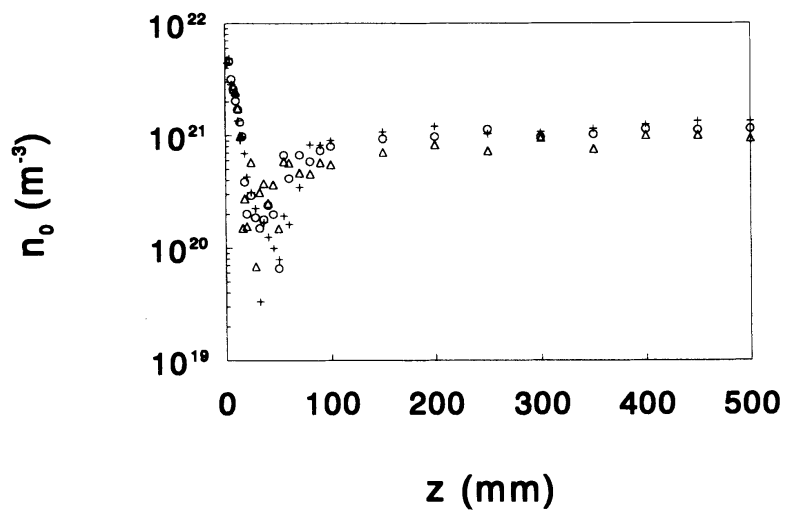

(b)

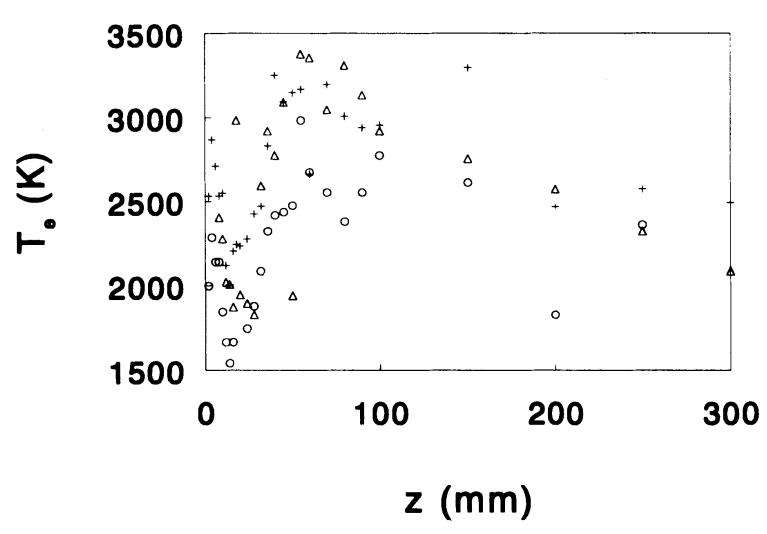

(c)

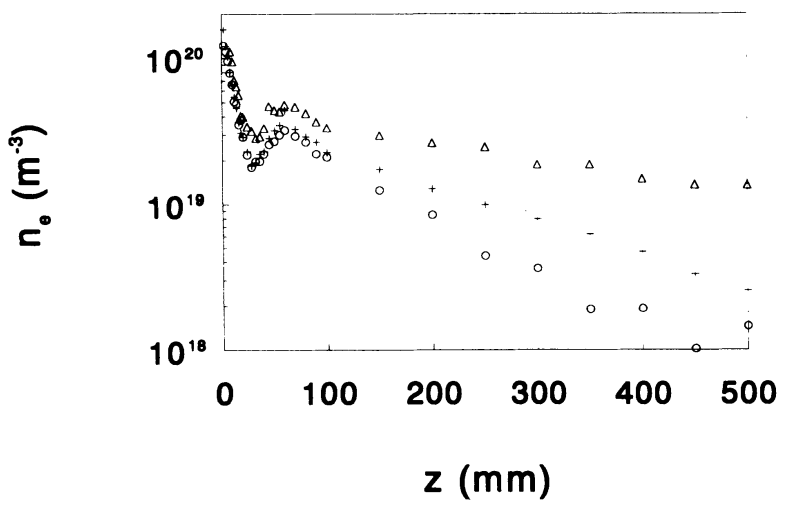

FIG. 4. The results from the Thomson-Rayleigh scattering measurements for the three conditions mentioned in the text. (a) The neutral density $n_{0}$ vs the axial position $z$; (b) the electron temperature $T_{e}$ vs $z$; (c) the electron density $n_{e}$ vs $z$. $\triangle$, pure argon;,$+ 0.7 \mathrm{vol} \% \mathrm{H}_{2} ; \bigcirc, 1.4 \mathrm{vol} \% \mathrm{H}_{2}$.

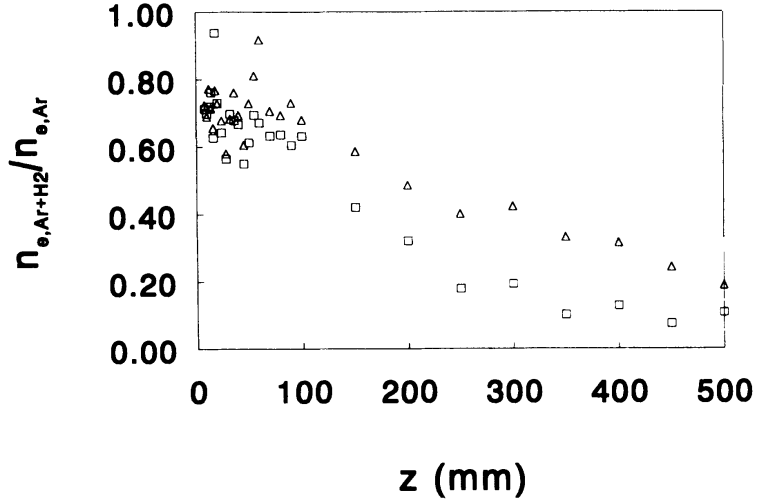

FIG. 5. The normalized electron density $n_{e, \mathrm{Ar}+\mathrm{H}_{2}} / n_{e, \mathrm{Ar}}$ vS the axial position $z$. Triangles denote 0.7 vol $\% \mathrm{H}_{2}$, squares denote 1.4 vol $\mathrm{H}_{2}$.

ing ionized species. The constant $C=\left(2 \pi m_{e} k / h^{2}\right)^{3 / 2}$, $u_{s}+$ is the ion partition function. The other symbols have their usual meaning: $k$ is Boltzmann's constant; $h$ is Planck's constant; $m_{e}$ is the electron rest mass; $T_{e}$ is the electron temperature; $E_{i}$ is the ionization potential of the species concerned; and $E_{p}$ is the excitation energy from the atom ground state of the level designated $p$. The lowering of the ionization potential due to the plasma environment is very small $(0.01 \mathrm{eV})$ and is neglected [11].

If we write Eq. (1) for both the hydrogen and the argon system, an expression for the ion concentration ratio can be obtained:

$\frac{n_{\mathrm{Ar}^{+}}}{n_{\mathrm{H}^{+}}}=\frac{\left(n_{p} / g_{p}\right)_{\mathrm{Ar}_{\mathrm{r}} u_{\mathrm{Ar}^{+}}}}{\left(n_{q} / g_{q}\right)_{\mathrm{H}} u_{\mathrm{H}^{+}}}\left(\frac{\left(E_{i}-E_{p}\right)_{\mathrm{Ar}}-\left(E_{i}-E_{q}\right)_{\mathrm{H}}}{k T_{e}}\right)$,

where the hydrogen excited level is designated $q$. When applying Eq. (2), our main interest is to make sure that the upper levels of the transitions chosen are in Saha equilibrium with their respective continua, i.e., that their population is governed by (1). An estimate of this "Saha region" for recombining plasmas can be obtained using $[11,12]$. In our case, with $n_{e} \approx 3 \times 10^{19} \mathrm{~m}^{-3}$ and $T_{e} \approx 0.20$ $\mathrm{eV}$, the region of Saha equilibrium extends to about 1.1 $\mathrm{eV}$ from the continuum. This leads us to use $\mathrm{H} \delta$ $\left(E_{i}-E_{q}=0.378 \mathrm{eV}\right)$ and $\operatorname{ArI} 518.8 \mathrm{~nm}\left(E_{i}-E_{p}=0.456\right.$ $\mathrm{eV}$ ). The accuracy of $n_{\mathrm{Ar}^{+}} / n_{\mathrm{H}^{+}}$is mainly determined by the accuracy of $n_{p}$ and $n_{q}$, as $T_{e}$ is known within $20 \%$.

Figure 9, then, gives the results of the calculations using Eq. (2): the $n_{\mathrm{Ar}^{+}} / n_{\mathrm{H}^{+}}$ratios for both conditions with hydrogen and for the three $z$ positions where measurements were taken. The corresponding absolute ion concentrations can be calculated using the principle of quasineutrality;

$$
n_{\mathrm{Ar}^{+}}+n_{\mathrm{H}^{+}}=n_{e},
$$

where we neglect doubly ionized species as well as molecular and negative ions as a first approximation. As 
$n_{\mathrm{Ar}^{+}} \gg n_{\mathrm{H}^{+}}$, in particular at the plasma axis (Fig. 9), we can actually take $n_{e} \approx n_{\mathrm{Ar}^{+}}$.

\section{Combined results}

We can look more closely at the presence of some kind of equilibrium between the different excited levels by combining the OES results with the Thomson-Rayleigh scattering data. To do so, we introduce the population

(a)

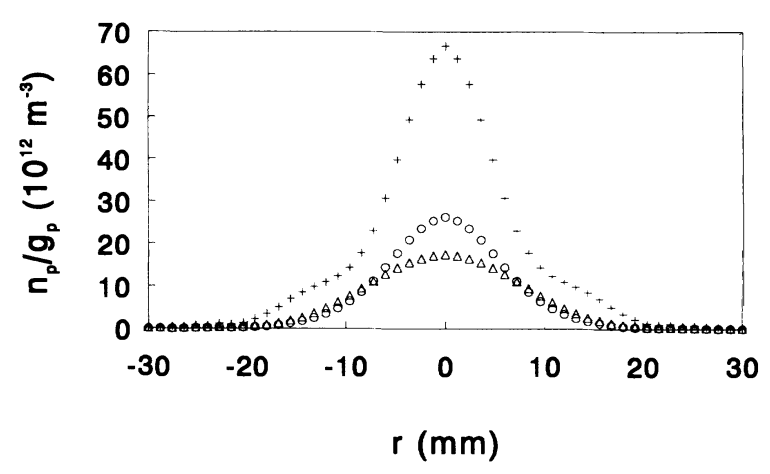

(b)

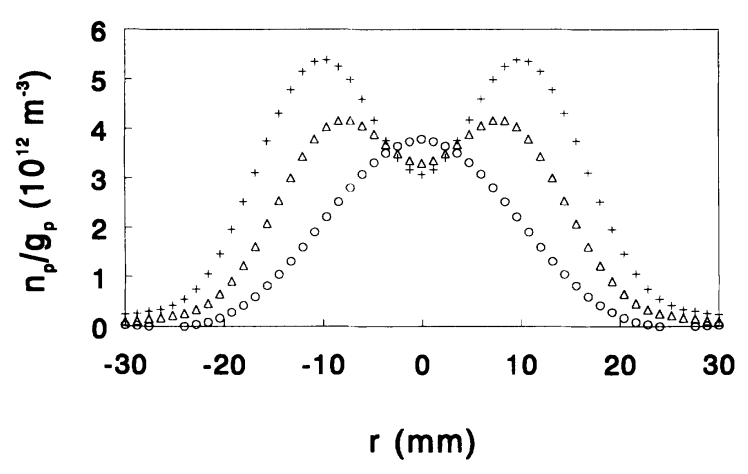

(c)

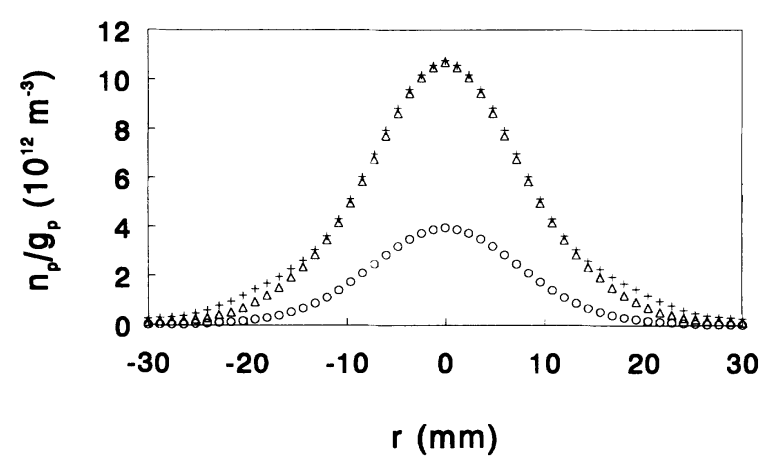

FIG. 6. Optical emission spectroscopy results: $\operatorname{Ar} 4 p^{\prime}\left[\frac{1}{2}\right]$ density (per statistical weight) radial profiles, for different hydrogen admixtures, at three $z$ positions: (a) $z=20 \mathrm{~mm}$; (b) $z=40$ $\mathrm{mm}$; and (c) $z=70 \mathrm{~mm}$. +, pure argon; $\triangle, 0.7$ vol \% $\mathrm{H}_{2} ; 0,1.4$ vol \% $\mathrm{H}_{2}$. factor $b_{p}$, which represents the absolute level population weighed against the level population according to Saha,

$$
b_{p}=\frac{n_{p}}{n_{p, \text { Saha }}},
$$

where $n_{p, \text { Saha }}$ is calculated from (1) using the measured values of $n_{e}$ and $T_{e}$ (Fig. 4). In the region of Saha equilibrium of course $b_{p}=1$.

For the case of a pure argon plasma, $b_{p}$ vs $E_{i}-E_{p}$ plots for all levels measured are presented at three $z$ positions. As can clearly be seen, $b_{p} \approx 1$ holds for highly excited lev-

(a)

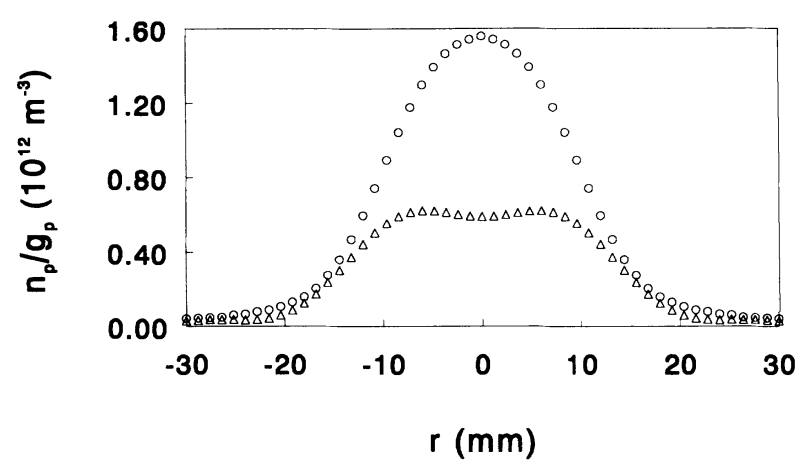

(b)

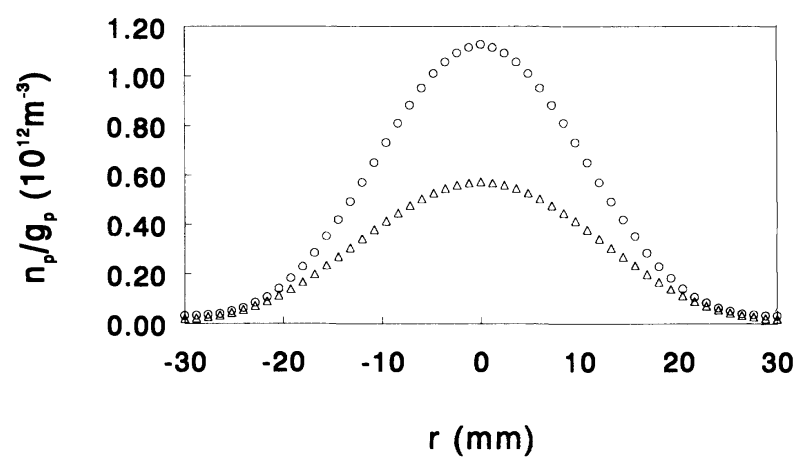

(c)

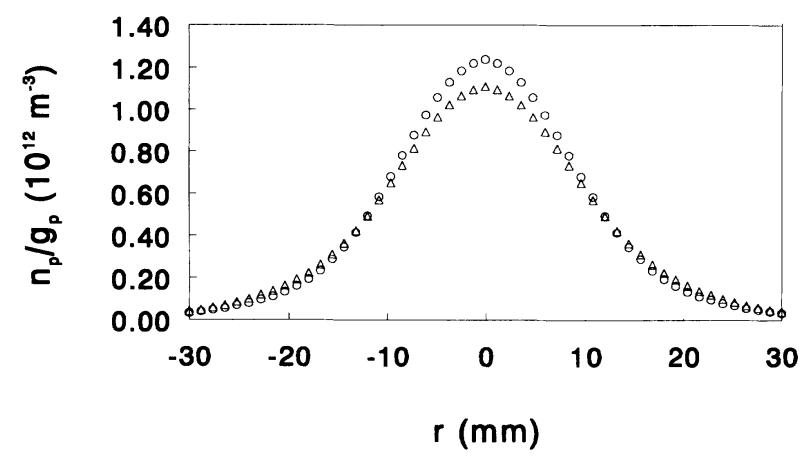

FIG. 7. OES results: $\mathbf{H}(p=3)$ density (per statistical weight) radial profiles for different hydrogen admixtures, at (a) $z=20$ $\mathrm{mm}$; (b) $z=40 \mathrm{~mm}$; and (c) $z=70 \mathrm{~mm}$. $\triangle, 0.7 \mathrm{vol} \% \mathrm{H}_{2} ; \bigcirc, 1.4$ vol \% $\mathrm{H}_{2}$. 
els $\left(E_{i}-E_{p} \leq 0.5 \mathrm{eV}\right)$ at all $z$ positions. This constitutes a secure test for measurements and calibrations, as these plots combine theory $\left(b_{p}=1\right.$ for highly excited states in recombining plasmas), Thomason scattering results $\left(n_{e}, T_{e}\right.$ values to calculate $b_{p}$,Saha $)$, and OES results $\left(n_{p}\right.$ measurements). The fact that $b_{p}$ approaches unity when $E_{i}-E_{p}$ approaches zero means that the Thomson and OES measurements are in good agreement with each other within the experimental errors.

Finally, then, the $b_{p}$ vs $E_{i}-E_{p}$ plots for the hydrogenargon mixtures are presented in Figs. 10 and 11 (all at

(a)

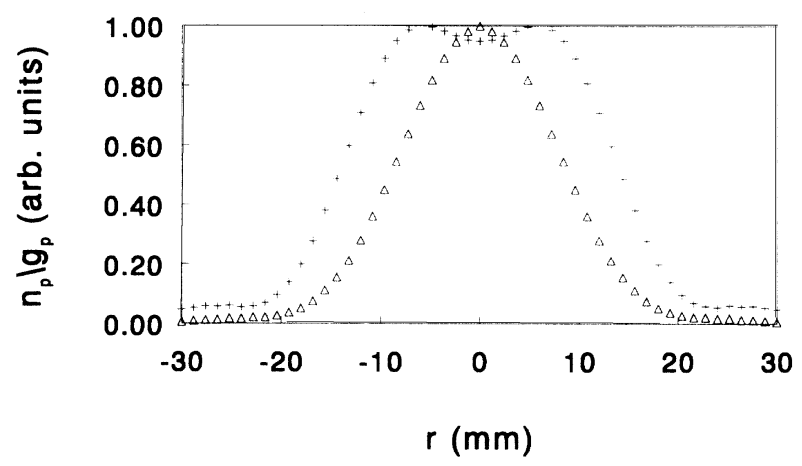

(b)

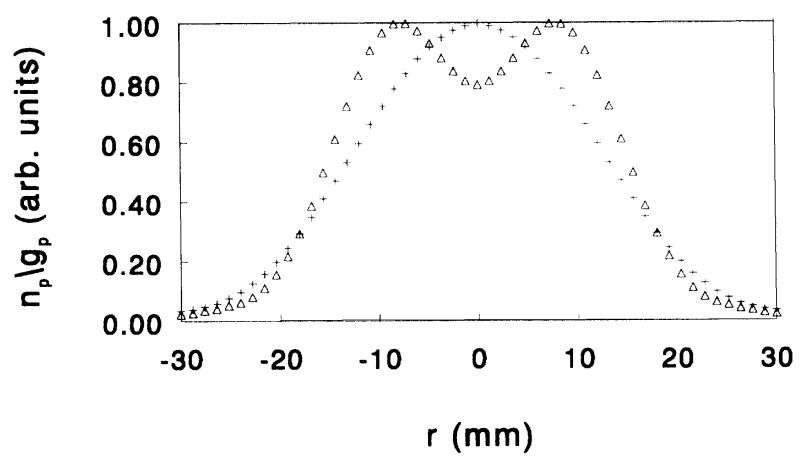

(c)

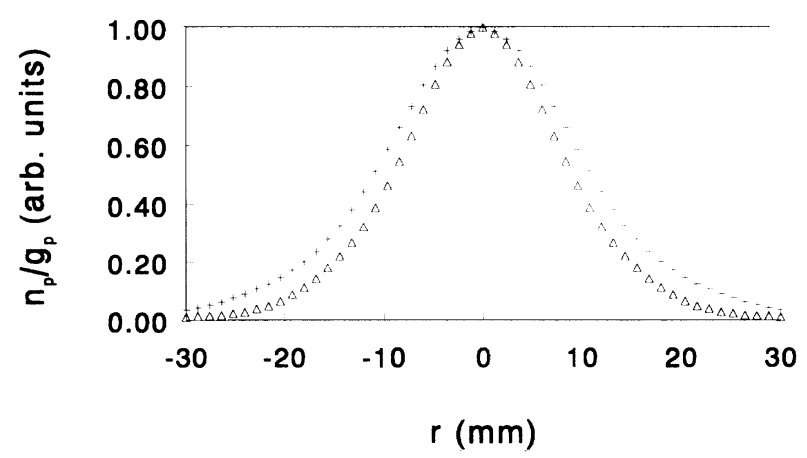

FIG. 8. OES results: $\operatorname{Ar}\left(4 p^{\prime}\right)$ and $H(p=3)$ level density (per statistical weight) radial profiles, normalized to 1 at $r=0$, for $0.7 \mathrm{vol} \% \mathrm{H}_{2}$ admixed, and at three $z$ positions: (a) $z=20 \mathrm{~mm}$; (b) $z=40 \mathrm{~mm}$; and (c) $z=70 \mathrm{~mm}$. $\triangle$, argon; + , hydrogen. The graphs for 1.4 vol \% are similar. $r=0$ ). The hydrogen and argon systems can be presented in a single frame, as $b_{p}$ is a universal quantity: the $b_{p}$ values for hydrogen and argon can actually be compared at the same $\left(E_{i}-E_{p}\right)$ value (i.e., at the same energy distance from their respective continuum). For the calculation of the hydrogen $b_{p}$ values, we used the data from Fig. 9 and Eq. (3) in order to obtain $n_{\mathrm{H}^{+}}$. For argon, we simply used $n_{\mathrm{Ar}^{+}}=n_{e}$ at the plasma axis.

\section{DISCUSSION}

\section{A. General}

In the present section, a qualitative model for the argon-hydrogen expanding plasma jet will be developed in order to understand the experimental findings. It is appropriate to start by presenting the general view, as supported by other measurements $[4,10,13-15]$, for this type of plasma.

Starting inside a pure argon cascaded arc, it has been established that $n_{e}, T_{e}$ and $T_{h}$ (the heavy particle temperature) are around $10^{22} \mathrm{~m}^{-3}, 1 \mathrm{eV}$, and $1 \mathrm{eV}$, respectively. The ionization degree is around $8 \%[3,7]$. With hydrogen added to the argon before it enters the arc, we assume this ionization degree to remain unchanged, at least for very low $\mathrm{H}_{2}$ seedings.

As a next step, we compare the dissociation energy of

(a)

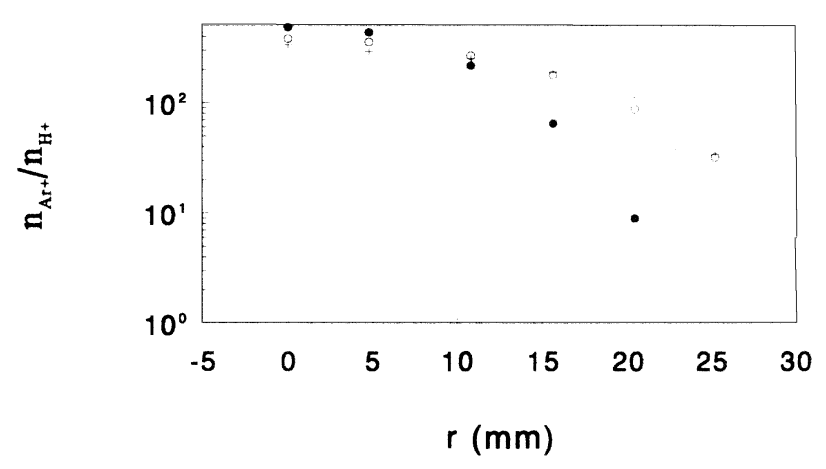

(b)

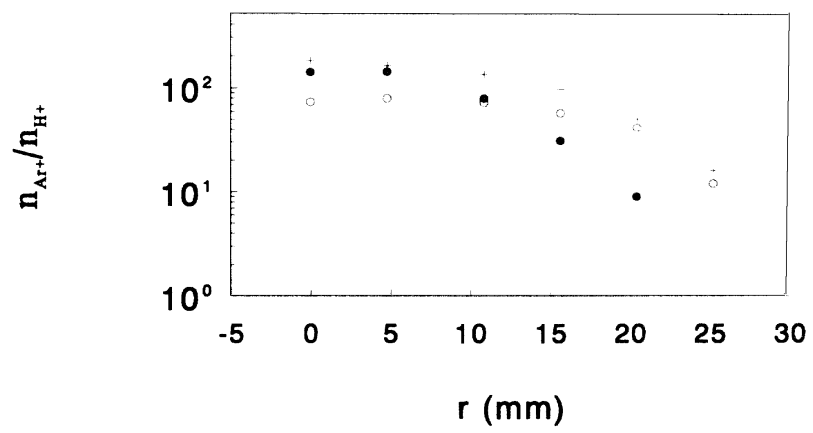

FIG. 9. The calculated ion concentration ratios $n_{\mathrm{Ar}^{+}} / n_{\mathrm{H}^{+}}$ vs $r$, at different $z$ positions: (a) $0.7 \mathrm{vol} \% \mathrm{H}_{2}$; (b) $1.4 \mathrm{vol} \% \mathrm{H}_{2}$. ๑, $z=20 \mathrm{~mm}$; $\bigcirc, z=40 \mathrm{~mm} ;+, z=70 \mathrm{~mm}$. 
$\mathrm{H}_{2}(4.4 \mathrm{eV})$, the ionization potential of $\mathrm{H}(13.6 \mathrm{eV})$, and the argon ionization potential $(15.8 \mathrm{eV})$. As in a pure argon arc, a substantial part of the particles is ionized, a comparison of the above potentials leads us to believe that with a small amount of hydrogen added, the hydrogen will be completely dissociated. The $n_{\mathrm{Ar}^{+}} / n_{\mathrm{H}^{+}}$ratio can be calculated using the Boltzmann equation:

$\frac{{ }^{n} \mathrm{Ar}^{+}}{{ }^{n} \mathrm{H}^{+}}=\frac{{ }^{n} \mathrm{Ar}}{n_{\mathrm{H}}} \frac{g_{\mathrm{Ar}}{ }^{+}}{g_{\mathrm{Ar}}} \frac{g_{\mathrm{H}}}{g_{\mathrm{H}^{+}}} \exp \left(\frac{13.6-15.8}{T_{e}(\mathrm{eV})}\right)$.

For a 0.7 vol $\% \mathrm{H}_{2}$ seed fraction (i.e., $1.4 \% \mathrm{H}$ particles)

(a)

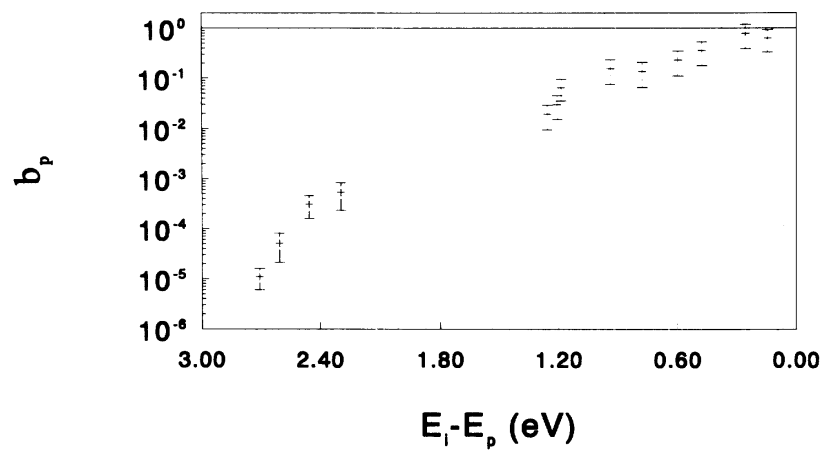

(b)

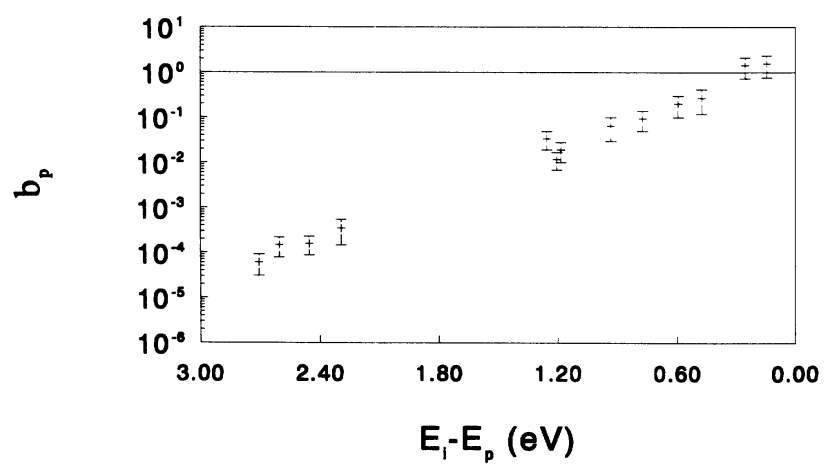

(c)

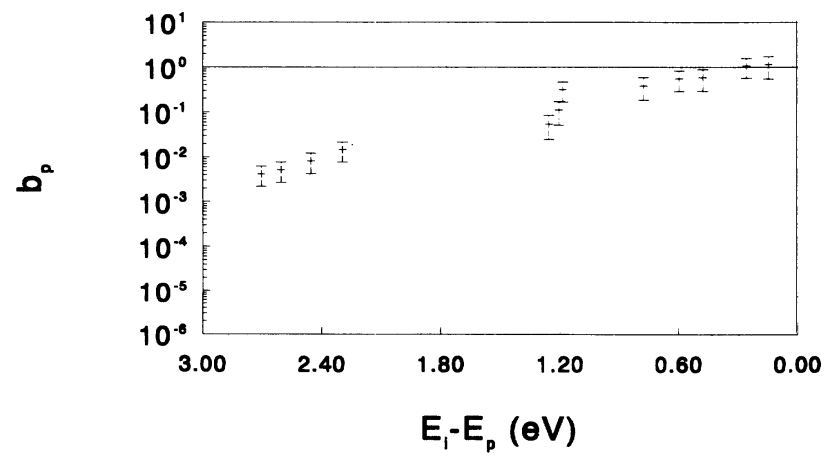

FIG. 10. The population factor $b_{p}$ vs $E_{i}-E_{p}$ for the expanding plasma jet in pure argon: (a) $z=20 \mathrm{~mm}$; (b) $z=40 \mathrm{~mm}$; and (c) $z=70 \mathrm{~mm}$. and $T_{e}=1 \mathrm{eV}$, Eq. (5) gives an ${ }_{\mathrm{Ar}^{+}} / n_{\mathrm{H}^{+}}$ratio (inside the cascaded arc) of approximately $(100 / 1.4) \times 12 \times 0.11$ $\approx 10^{2}$. Thus, very few $\mathrm{H}^{+}$ions are produced in the arc if we assume the ionization degree to be roughly the same as in the pure argon case.

With our assumption that only $\mathrm{Ar}^{+}, \mathrm{Ar}, \mathrm{H}^{+}, \mathrm{H}$, and $e^{-}$particles leave the arc, the extremely enhanced recombination with hydrogen added (Fig. 4) is difficult to explain, as ordinary three-particle recombination is far too slow at the densities and temperatures present [4]. Therefore, we are forced to consider the influence of the stainless-steel vacuum vessel in which the expansion takes place (length $3 \mathrm{~m}$, diameter $0.36 \mathrm{~m}$ ). It is a well-known

(a)

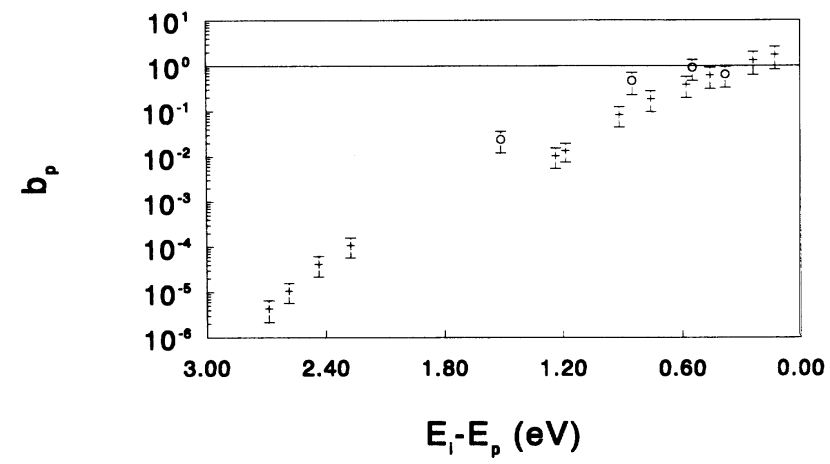

(b)

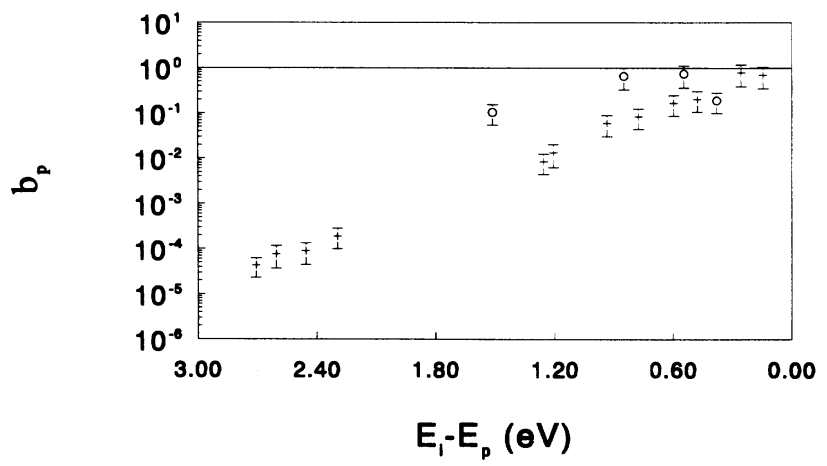

(c)

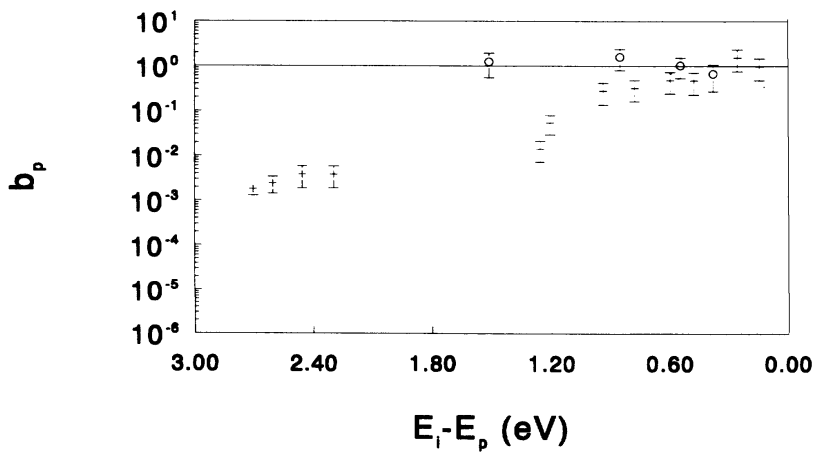

FIG. 11. The population factor $b_{p}$ vs $E_{i}-E_{p}$ for 0.7 vol $\%$ $\mathrm{H}_{2}$ admixed: (a) $z=20 \mathrm{~mm}$; (b) $z=40 \mathrm{~mm}$; and (c) $z=70 \mathrm{~mm}$. + , argon; $O$, hydrogen. 
fact that stainless steel constitutes a perfect surface for association of hydrogen atoms. In our case (Fig. 12 gives a sketch), after recombination in the subsonic part of the expansion, hydrogen atoms will reach the walls: the vessel walls may well be saturated with hydrogen. Association and subsequent desorption of hydrogen molecules could be responsible for a reentry flow of hydrogen molecules (rotationally and vibrationally excited) into the expanding plasma, in combination with a recirculation pattern, which may be present within the vessel.

It is this reentry flow of wall-associated hydrogen molecules that is thought to be responsible for the fast ionization loss in the expansion. The following is the proposed reaction mechanism $[10,14,15]$ :

$$
\mathrm{Ar}^{+}+\mathrm{H}_{2}(\nu=0) \rightarrow \mathrm{ArH}^{+}+\mathrm{H}
$$

followed by

$$
\mathrm{ArH}^{+}+e^{-} \rightarrow \mathrm{Ar}+\mathrm{H}^{*} .
$$

The second (dissociative recombination) step is known to be very fast (rate constant around $10^{-13} \mathrm{~m}^{3} \mathrm{~s}^{-1}$ ), while the first step has a rate constant lower by a factor of about $10^{2}$ at the present conditions $\left(K=1.1 \times 10^{-15}\right.$ $\left.\mathrm{m}^{3} \mathrm{~s}^{-1},[16]\right)$. In principle, $\mathrm{Ar}^{+}$can also react with $\mathrm{H}_{2}$ to form $\mathrm{H}_{2}{ }^{+}$,

$$
\mathrm{Ar}^{+}+\mathrm{H}_{2} \rightarrow \mathrm{Ar}+\mathrm{H}_{2}^{+}
$$

followed by dissociative recombination $[14,15]$. This reaction, however, has been shown to be of little importance for energies below $0.5 \mathrm{eV}$ [17]; at low energies, reaction (6) dominates.

Another reaction that may occur involves vibrationally excited hydrogen molecules and hydrogen ions,

$$
\mathrm{H}^{+}+\mathrm{H}_{2}(v>4) \rightarrow \mathrm{H}_{2}{ }^{+}+\mathrm{H},
$$

followed by

$$
\mathrm{H}_{2}^{+}+e^{-} \rightarrow \mathrm{H}+\mathrm{H}^{*} \text {. }
$$

The reaction rates for (10) and (7) are about equal. The reaction rate for (9) is about $2.5 \times 10^{-15} \mathrm{~m}^{3} \mathrm{~s}^{-1}$ [14]. Note that both in (6) and (10), the formed hydrogen appears in an excited state, most probably $p=2$ or 3 .

\section{B. Thomson-Rayleigh scattering}

From the Thomson-Rayleigh measurements, we can actually make an estimate of the amount of (reentrant)

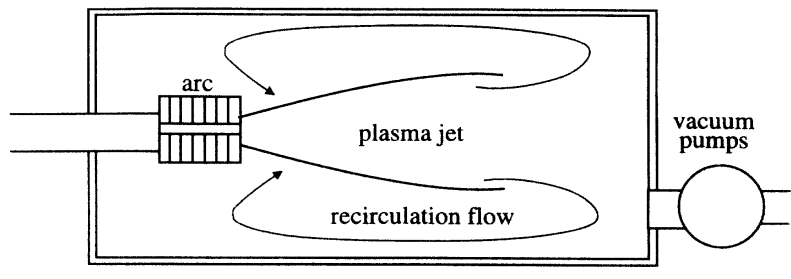

FIG. 12. A sketch of the recirculation pattern within the vacuum vessel. The recirculation flow is responsible for the transport of the $\mathrm{H}_{2}$ from the vessel walls into the plasma jet. hydrogen molecules. To do so, we assume that reactions (6) and (7) constitute the dominant process in the ionization loss as seen in Fig. 4(c). We describe this dominant loss term in the electron mass balance as follows:

$$
\left(\frac{\partial n_{e}}{\partial t}\right)_{\text {Eqs. (6) and (7) }}=-n_{\mathrm{Ar}^{+}} n_{\mathrm{H}_{2}} K_{\mathrm{ArH}^{+}} \text {, }
$$

as the first step (6) determines the reaction speed. If we assume for the moment, $n_{\mathrm{Ar}^{+}} \approx n_{e}$ and $n_{\mathrm{H}_{2}}$ constant along the $z$ axis, (11) is integrated easily,

$$
\ln \left(\frac{n_{e, t}}{n_{e, t=0}}\right)=-n_{\mathrm{H}_{2}} K_{\mathrm{ArH}^{+}} t .
$$

In (12), $t=0$ corresponds to $z=70 \mathrm{~mm}$, whereas $t=t$ corresponds to $z=500 \mathrm{~mm}$ (see Fig. 4). Thus, $t=\Delta z / v_{z}$, with $v_{z}$ the axial velocity after the shock; $v_{z} \approx 600 \mathrm{~m} / \mathrm{s}$. We take $K_{\mathrm{ArH}^{+}}=1.1 \times 10^{-15} \mathrm{~m}^{3} \mathrm{~s}^{-1}$. If we insert the measured quantities for a 0.7 vol $\%$ seed fraction $n_{e, t=0}=3 \times 10^{19} \mathrm{~m}^{-3}, n_{e, t=t}=3 \times 10^{18} \mathrm{~m}^{-3}, \Delta z \approx 0.430$ $\mathrm{m}$, we obtain an estimate for $n_{\mathrm{H}_{2}}$,

$$
n_{\mathrm{H}_{2}} \approx 3 \times 10^{18} \mathrm{~m}^{-3} \text {. }
$$

So far, we have neglected any influence of reactions (9) and (10), even though the rate of the slower of these two, (9), exceeds that of (6) by a factor of about 2 . However, the $\mathrm{H}^{+}$concentration is smaller than the $\mathrm{Ar}^{+}$concentration by a factor of 10-500. Furthermore, a hydrogen molecule must be vibrationally excited in order to participate in reaction (9). Thus, even though the rate constant of (9) is large, the contribution of reactions (9) and (10) to the ionization loss can be neglected in our plasma.

\section{Optical emission spectroscopy}

The most crucial point in the discussion of the OES measurements is the origin of a certain level's population. In principle, this population is a result of excitation from lower levels and/or recombination from higher ones. In cases where $T_{e}$ is very low, i.e., in the $0.2-\mathrm{eV}$ range, only three-particle recombination is important (for atomic plasmas). In a pure argon expanding plasma jet, the levels are populated by the destruction of $\mathrm{Ar}^{+}$ions [4]. From Eq. (1), it can be shown that this results in a level population roughly proportional to $n_{e}^{2}$. Thus, in a sense, we are "looking at" the $\mathrm{Ar}^{+}$population by examining the atomic state distribution function in the Ar I system.

In our case, where two ion species $\left(\mathrm{Ar}^{+}\right.$and $\mathrm{H}^{+}$, in a ratio of about 100:1) leave the arc, the situation is more complex. We shall confine ourselves to the view, that Ar I excited states are populated mainly through threeparticle recombination, i.e., we leave out processes such as excitation exchange for the moment. As three-particle recombination is proportional to $T_{e}^{-9 / 2}$, higher line intensities are to be expected at $z=20 \mathrm{~mm}$, where $T_{e}$ is low [Fig. 4(b)]. This is reflected in the behavior of the $\left(4 p^{\prime}\right)$ levels, shown in Fig. 6.

The hydrogen excited level population may also arise from dielectronic recombination, but in this case an extra source term almost certainly comes in: the dissociative 
recombination in (7) and (10), ending in hydrogen excited states $p=2,3$. The $\mathrm{H}_{\mathrm{I}} 636.5 \mathrm{~nm}$ emission probably arises due to a combination of the two processes: three-particle recombination mainly at the plasma axis and dissociative recombination "wings" at the edges, where the reentrant $\mathrm{H}_{2}$ molecules are most abundant. This is reflected in the fact that the $p=3$ radial profiles are broader than the corresponding $\mathrm{Ar}_{4 p^{\prime}}$ profiles (Fig. 8), especially at $z=20$ $\mathrm{mm}$.

One can attempt to explain the changing of the shock behavior as reflected in the $\mathrm{Ar}\left(4 p^{\prime}\right)$ emission [Fig. 6(b)] at $z=40 \mathrm{~mm}$ within the qualitative model. At the plasma axis, the $\left(4 p^{\prime}\right)$ density is roughly constant for different hydrogen admixtures, but the characteristic ridges at $r \approx \pm 20 \mathrm{~mm}$ (in pure argon) are smoothened and eventually disappear. This can be viewed analogously to the electron density decrease in axial direction, as seen in Fig. 4(b). If we still assume that Ar I emission represents the $\mathrm{Ar}^{+}$population, as stated above, the disappearance of the shock ridges may be due to reactions (6) and (7) under the influence of radially entering $\mathrm{H}_{2}$ molecules. Figure 13 gives a sketch of the hypothetical analogy: the Ar I emission at the edges of the beam decreases due to the loss of argon ions.

The fact that the hydrogen system has an extra input can also be seen in Figs. 10 and 11: all $H$ levels have higher to much higher $b_{p}$ values (up to a factor $10^{2}$ ) compared to argon (at a certain $E_{i}-E_{p}$ ). Note the fact that even $p=3$ has $b_{p} \approx 1$ at $z=70 \mathrm{~mm}$ for $E_{i}-E_{p}=1.511 \mathrm{eV}$. The overpopulation of $p=2,3$ states due to dissociative recombination could be smoothened over the other levels by electron collisions at $T_{e} \approx 0.2 \mathrm{eV}$.

At $z=70 \mathrm{~mm}$, the plasma is almost homogeneous, as illustrated by Fig. 8(c): the hydrogen and argon profiles are almost equally wide-a substantial difference with $z=20 \mathrm{~mm}$, indeed. This may point to the reentrant hydrogen molecules being absorbed by the beam before the stationary shock front at $z \approx 40-70 \mathrm{~mm}$. As can be seen from Figs. 10 and 11, the atomic level distribution function is closest to Saha equilibrium at $z=70 \mathrm{~mm}$, because $T_{e}$ is higher than at the other two $z$ positions considered here [Fig. 4(b)]. The $n_{\mathrm{Ar}^{+}} / n_{\mathrm{H}^{+}}$ratio turns out to be of the right order of magnitude [compare the outcome of Eq. (5) to Fig. 9 for a 0.7 vol \% seed in]. However, it is higher by a factor of about 5. Some extra $\mathrm{H}^{+}$loss can be explained through gasdynamical reasoning based on the fact that hydrogen ions are lighter than argon ions by a factor of 40. This should be reflected in a much faster radial and axial expansion (fact $\sqrt{40} \approx 6$ ), within the limits of quasineutrality. This results in a larger $n_{\mathrm{Ar}^{+}} / n_{\mathrm{H}^{+}}$ratio (factor 6).
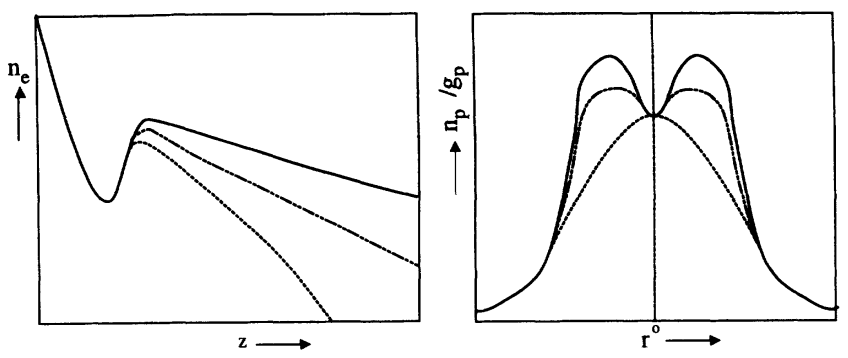

FIG. 13. The disappearance of the shock ridges in the AR I $\left(4 p^{\prime}\right)$ radial profiles (see Fig. 6) is the radial analogy of the electron density decrease in axial direction [see Fig. 4(c)], as a consequence of hydrogen molecules reentering the plasma jet.

\section{Conclusions}

The $n_{0}$ vs $z$ profile shows that the gas dynamical expansion in axial direction is not heavily influenced by adding a small amount of hydrogen to the plasma.

The $n_{e}$ axial profile, however, is severely changed by the addition of hydrogen, most probably because of a reentry flow of hydrogen molecules. These molecules are formed at the vessel walls and could trigger a very effective recombination channel (6) and (7).

The broad hydrogen emission profiles, especially at $z=20 \mathrm{~mm}$, can be explained by the occurrence of this reaction at the edges of the plasma. A different expansion behavior of hydrogen may also induce broader radial profiles.

The smoothening of the shock ridges in the argon ( $\left.4 p^{\prime}\right)$ profiles could be caused by reactions (6) and (7) occurring at the edges of the plasma: the $\left(4 p^{\prime}\right)$ level density at the plasma axis is almost unaffected.

The ${ }_{\mathrm{Ar}^{+}} / n_{\mathrm{H}^{+}}$concentration ratios are larger than expected (following the basic arc picture) by a factor of about 5 . This can be explained by the fact that hydrogen ions are much lighter than their argon counterparts.

The fact, that highly excited levels in the Ar I system approach $b_{p}=1$ (in all three conditions) is a solid test for both Thomson-Rayleigh and OES measurements.

\section{ACKNOWLEDGMENTS}

We would like to thank M.J.F. van de Sande and H.M.M. de Jong for their skillful technical assistance during the measurements.
[1] A. T. M. Wilbers, G. J. Meeusen, M. Haverlag, G. M. W. Kroesen, and D. C. Schram, Thin Solid Films 204, 59 (1991).

[2] J. J. Beulens, A. J. M. Buuron, L. A. Bisschops, A. B. M. Hüsken, G. M. W. Kroesen, G. J. Meeusen, C. J. Timmermans, A. T. M. Wilbers, and D. C. Schram, J. Phys. (Paris) Colloq. 51, C5-361 (1990).

[3] P. K. Bachmann, J. J. Beulens, G. M. W. Kroesen, H.
Lydtin, D. C. Schram, and D. U. Wiechert, Surf. Modif. Technol. 3, 69 (1989).

[4] M. C. M. van de Sanden, Ph. D. thesis, Eindhoven University of Technology, 1991 (unpublished).

[5] M. C. M. van de Sanden, J. M. de Regt, G. M. Jansen, J. A. M. van der Mullen, D. C. Schram, and B. van der Sijde, Rev. Sci. Instrum. 63, 3369 (1992).

[6] R. F. G. Meulenbroecks, P. A. A. van der Heijden, M. C. 
M. van der Sanden, and D. C. Schram, J. Appl. Phys. 75, 2775 (1994).

[7] G. M. W. Kroesen, D. C. Schram, and J. C. M. de Haas, Plasma Chem. Plasma Proc. 10, 531 (1990).

[8] C. A. Kak and M. Slaney, Principles of Computerized Tomographic Imaging (IEEE, New York, 1988).

[9] W. L. Wiese, M. W. Smith, and B. M. Miles, Atomic Transition Probabilities, Natl. Stand. Ref. Data Ser., Natl. Bur. Stand. (U.S.) Circ. No. 22 (U.S. GPO, Washington, D.C. 1969).

[10] G. J. Meeusen, E. A. Ershov-Pavlov, R. F. G. Meulenbroeks, M. C. M. van de Sanden, and D. C. Schram, J. Appl. Phys. 71, 4156 (1992).

[11] L. M. Biberman, V. S. Vorob'ev, and I. T. Yakubov, Kinetics of Nonequilibrium Low-Temperature Plasmas
(Plenum, New York, 1987).

[12] J. A. M. van der Mullen, Phys. Rep. 191, 161 (1989).

[13] R. F. G. Meulenbroeks, D. C. Schram, L. J. M. Jaegers, and M. C. M. van de Sanden, Phys. Rev. Lett. 69, 1379 (1992).

[14] M. J. de Graaf, R. P. Dahiya, J. L. Jauberteau, F. J. de Hoog, M. J. F. van de Sande, and D. C. Schram, J. Phys. (Paris) Colloq. 51, C5-387 (1990).

[15] M. J. de Graaf, R. Severens, R. P. Dahiya, M. C. M. van de Sanden, and D. C. Schram, Phys. Rev. E 48, 2098 (1993).

[16] K. M. Ervin and P. B. Armentrout, J. Chem. Phys. 83, 166 (1895).

[17] Eric A. Gislason and Gérard Parlant, J. Chem. Phys. 94, 6598 (1991). 